\title{
An integrated optimized hybrid intensity modeled brain tumor image segmentation using artificial bee colony algorithm
}

\author{
Mubeena V.* \\ Guest Lecturer, Department of Computer Science, Farook College, Feroke, Kozhikode, Kerala
}

\section{(C2018 ACCENTS}

\begin{abstract}
Brain magnetic resonance (MR) images are very significant in early detection and diagnosis of the brain tumor treatment. In order to improve the efficiency and accuracy of the brain tumor segmentation in MR images, an integrated hybrid segmentation approach is proposed. This approach is developed by integrating the features of local independent projection based classification (LIPC), partitioning around the medoids (PAM) and enhanced fuzzy c- means (EFCM) approach that is employed for the determination of the cluster centers that classify MR images accurately based on independent projections of the tumor cells. This segmentation approach is considered as classification problem. The classification accuracy is improved by incorporating additional contextual features for the tumor cells. The segmented image from the hybrid method may have some healthy cells included in the tumor regions due to tumor mass effect. Thus an improved intensity modeling is proposed to eliminate the tumor mass effect and also to improve the accuracy in classification. The above work utilizes the artificial bee colony $(A B C)$ algorithm for the improvement in the segmentation of tumor cells below the surface area. Here, the tumor regions are clustered into colonies and each colony is enhanced to a solution. The colonies are combined with the synthetic data of the images. The ABC technique enables the segmentation even in deeper layers by partitioning the tumor images around the lesions that makes the proposed system to better result in efficient detection and segmentation of brain tumor from the MR images.
\end{abstract}

\section{Keywords}

Brain MR images, LIPC, EFCM, ABC algorithm.

\section{Introduction}

The brain tumor is one of the complex diseases as it is considered difficult for detection and diagnosis. Magnetic resonance imaging (MRI) is used to capture the current structure of human brain image. Though the MRI images are effectively used for the detection of brain structure, the processing of the images is a tedious task. The manual processing of the brain images is time consuming and also has highly reduced accuracy of brain image analysis. The manual detection also tends to produce corruption or deterioration of the brain images which is the topmost problem. Hence, the automatic processing of the brain images has become the need of the hour. But, even the automatic processing methods are still under various hurdles as it is in the beginning of the evolution phase. Thus, the automatic brain tumor detection or the identification of the tumor cells from the group of healthy cells becomes an interesting topic of research.

\footnotetext{
*Author for correspondence
}

Various techniques have been presented in the recent years to support the automatic detection of the brain tumor. Image segmentation is an important task in the processing of brain images. The brain tumor image segmentation methods such as compression based methods, histogram based methods, edge detection, clustering methods, region growing methods, graph partitioning methods and watershed \& threshold methods [1,2] have been presented by various researchers to improve the accuracy of segmentation. The diagnosis of the brain tumor depends on the accurate classification of the MRI brain images.

Hence in this article, an improved intensity modeling is proposed to eliminate the tumor mass effect and also to improve the accuracy in classification. This technique utilizes $\mathrm{ABC}$ algorithm to improve the segmentation of tumor cells below the surface area. The tumor regions are clustered into colonies and each colony is enhanced to a solution. The colonies are combined with the synthetic data of the images. Thus, the segmentation and detection of tumor cells is enhanced by the proposed method. 


\section{Related works}

In this section, the works related to the brain tumor detection techniques are discussed in detail. Jason $\mathbf{J}$. Corso et al. [3] presented a new method for automatic segmentation of heterogeneous image data that is applicable in any case that distinct feature distributions can be learned for the heterogeneous regions. The method combines two of the most effective approaches to segmentation. The first approach uses class models to explicitly represent the different heterogeneous processes. The tasks of segmentation and classification are solved jointly by computing solutions that maximize a posterior distribution that has been learned from training data. The second approach is based on the concept of graph cuts in which affinity-based methods, the input data induces a sparse graph, and each edge in the graph is given an affinity measurement that characterizes the similarity of the two neighboring nodes in some predefined feature space.

Iftekharuddin et al. [4] proposed a method for detecting the brain tumor by fusing two novel texture features along with intensity features. The two features involves piecewise-triangular-prism-surfacearea (PTPSA) algorithm and fractional Brownian motion (fBm) framework for the extraction of fractal and wavelet features using both fractal and wavelet analyses. The MR image modalities such as T1 (gadolinium-enhanced), T2 and fluid-attenuated inversion-recovery (FLAIR) are exploited and the features are fused using self-organizing map (SOM). Though the approach improves the detection accuracy of the brain tumor, the time complexity may become a major issue at critical stages.

Szwarc et al. [5] presented a multi-stage automatic method for the effective detection of the brain tumor. The method also performs neo-vasculature assessment for detecting the formation of new blood vessels to serve the tumor cells other than the normal cells. The brain symmetry is exploited from the MRI analysis. Then the intracranial structures and the region-of-interest (ROI) along with the contrastenhanced lesions are detected on the basis of T1weighted (T1W) differential images. This enables better detection of the lesions without many false positives. But, still the detection of the bi-hemisphere lesion and extensive edema regions require further improvement.

Segmenting the MRI images is an important process in brain tumor detection. Aslam et al. [6] presented improved edge detection algorithm for segmenting brain tumor. The presented algorithm combines the sobel method with image dependent thresholding method, and finds different regions using closed contour algorithm after which the tumors are extracted from the MRI images using intensity information within closed contour. The only drawback with the approach is the thickness of the boundary lines of the regions that reduce the segmentation performance.

Nabizadeh et al. [7] proposed an automatic algorithm for the detection and segmentation of brain stroke and tumor lesion with the help of single-spectral MRI. The proposed Histogram-based Gravitational Optimization Algorithm (HGOA) was a novel intensity-based segmentation technique, where the enhanced gravitational optimization algorithm was applied on histogram analysis results. The convergence criteria and the mathematical description of the developed optimization algorithm were presented. The brain was segmented into different number of regions using this proposed algorithm, then labeled as lesion or healthy. The results provided us that the Ischemic stroke and tumor lesions were segmented with $91.5 \%$ and $88.1 \%$ accuracy. But, the proposed system has high computational burden if the higher recognition rate is required.

Mekhmoukh and Mokrani [8] proposed a segmentation method for image that relies on the particle swarm optimization (PSO) and outlier rejection combined with level set. A traditional approach called FCM clustering algorithm was sensitive to the outlier and the spatial information was not integrated in the image. In order to outlier rejection and also reduce the noise sensitivity, an extended FCM algorithm for image segmentation was proposed. In FCM algorithm the initial cluster centers were chosen by PSO algorithm, then optimally. Spatial neighborhood information was taken into consideration and used to reduce the cost function to set the level set functions. But the proposed algorithm do not adapt to data driven terms.

Kanasa et al. [9] proposed an automated method for segmenting neoplastic and healthy brain tissue in multi parametric MR images. The proposed method had outlier detection, intensity-based clustering, that was of low computational cost and also had an automated nature of detection. The outlier detection was based on global intensity modeling in the first step, and then a modification of the random walker algorithm was employed for local intensity variation. 
The scheme required only routine MRI. However, it is not suitable for non-enhancing tumors and necrosis.

Forouzanfar et al. [10] investigated the potential of genetic algorithms (GAs) and PSO for determining the optimum value of degree of attraction. The GAs gives a near optimal solution but not an exact solution, while PSO provide optimal solution. This work proposed a hybrid GAs/PSO method to determine the optimum degree of attraction. The results showed the improvements in segmentation of the proposed method compared to other FCM-based methods. The results of segmentation are best only when best selection operator of the PSO algorithm is chosen.

Shanthakumar and Ganeshkumar [11] proposed a technique called adaptive neuro fuzzy inference system (ANFIS) for the automatic seed point selection. In this work the intensity of the pixel is independent of the tumor type. Automatic tumor segmentation and the features are extracted from the brain image and sent to train a classifier. Based on the features extracted from the brain images, the proposed ANFIS classifier separates brain tumors as benign or malignant. In the final stage of processing, morphological functions are used to extract the brain tumor region. Clear difference of brain abnormalities from normal brain tissue was provided by the proposed method but computationally expensive.

Ain et al. [12] proposed a multi-stage system for brain tumor diagnosis and tumor region extraction. Initially, a noise removal was done on the brain MR images. Extraction of texture features are done from these de-noised brain MR images. Next, ensemble based support vector machine (SVM) classification was used to classify the images based on the features. Then, it extracted tumor region from the images using multi-step segmentation, where the first step was skull removal and extraction of the brain region. Then, the tumor region was separated from normal brain cells using proposed FCM clustering. Tumor type is not known using this technique.

Rajendran and Dhanasekaran [13] proposed a method which combined region based fuzzy clustering and deformable model for the segmentation of the tumor region in the MRI images. Region based fuzzy clustering was used for initial segmentation of tumor and then it was utilized to provide initial contour for deformable model and then the final contour for exact tumor boundary. This was used for final segmentation using gradient vector field as an external force field. Results showed that this method is more accurate and robust for brain tumor segmentation and also consumes more complexity in time.

Gilanie et al. [14] proposed a new method for extracting the object from T2 weighted brain MR images. It was based on histogram processing for gradient calculation and utilized the histogram filtering technique for the purpose pre-processing of the images. The areas such as Gray Matter (GM), White Matter (WM), and CerebroSpinal Fluid (CSF) of the brain were extracted from 2D and 3D images efficiently. Proposed method was successfully implemented on human brain MR images in clinical environment, but the technique lack high accuracy and precision.

Wu et al. [15] discussed about the drawbacks of most of the segmentation methods, especially in large data sets. In order to overcome such problems, a new method was developed where multimodal MR images were segmented into super pixels using algorithms. The features were extracted from the super pixels by multi-level Gabor wavelet filters. A SVM model and affinity metric model for tumors were trained. Conditional random field theory was applied to segment the tumor based on the output of the SVM and spatial affinity models to ensure smoothness. At last labeling noise was removed by the structural knowledge. But the challenge of data imbalance was present as a drawback.

Gaonkar et al. [16] proposed segmentation method based on the geodesic distance transform to segment 54 brain tumors which was semiautomatic. The brain metastases, meningiomas and glioblastomas were segmented. Tumor segmentations obtained using manual and automatic methods were compared by the parameter called dice measure of overlap. The proposed method addressed a significant need in the field of neuro-oncology that obtained accurate and reproducible tumor volumes without the need for manual segmentation. The proposed had a drawback that it required manual initialization of the segmentation process.

Joshi et al. [17] proposed a two stage segmentation where initially a Gabor filter banks generated using different frequencies and orientations are applied. ROI was identified from the filtered image and iterative contour detection method was applied to detect different objects in the image. The 
segmentation was achieved by discrete gray level sets and the tumor region was extracted by threshold segmentation. Simulation results were obtained based on the shape and size for the detection and analysis of the brain tumor. Texture and statistical features were not included.

Hamamci et al. [18] proposed seeded tumor segmentation method based on cellular automata (CA). This process is performed on the contrast enhanced T1-W MR images. The proposed work standardized the volume-of-interest (VOI) and seed selection. Initially, CA-based segmentation was connected to the graph-theoretic methods to solve the shortest path problem. The state transition function of the CA was modified to calculate the exact shortest path solution. A sensitivity parameter was introduced for its adaptation to the heterogeneous tumor segmentation problem to impose spatial smoothness. Also an algorithm based on CA was presented to differentiate necrotic and enhancing tumor tissue content for a detailed assessment of radiation therapy response. But, the proposed system fails in case of low intensity contrasts.

Menon and Ramakrishna [19] proposed an MRI Brain Image segmentation method which was based on ABC algorithm and FCM algorithm. Threshold estimation was used to search the value in continuous gray scale interval. Value of optimal threshold is found by $\mathrm{ABC}$ algorithm. An efficient fitness function for $\mathrm{ABC}$ algorithm is obtained when the original image is decomposed using the discrete wavelet transforms (DWT). Noise reduction was performed to the image, and the filtered image was reconstructed with low-frequency components. The FCM algorithm was used to cluster the segmented image that identified the brain tumor.

Jiang et al. [20] proposed a technique for the construction of a graph by studying the populationand patient-specific feature sets of the multimodal MR images. This is performed by using the graphcut for a final segmentation. Each pixel has a probability that defines whether it belongs to the foreground and the background were estimated by global and custom classifiers which are trained through learning population- and patient-specific feature sets, respectively. The proposed method showed improvement in the results when compared with other approaches. But, the proposed system performance is degraded when there is overlap of the voxel intensities in the tumor and normal tissues.
Koleya et al. [21] proposed a novel approach of characterization and delineation of four different types of brain tumors from magnetic resonance images. This was done in post Contrast T1-W sequence in order to improve the diagnostic accuracy. Thus, an integrated framework of identification and extraction of tumor region and quantification of histogram was proposed. The Rough entropy based thresholding was presented for delineation of tumor region. Quantitative validation and comparison with existing methods proved the efficiency and applicability of proposed segmentation approach. In the next stage, random forest (RF), an ensemble learning scheme was implemented, for accurate prediction of the class label of a given input. The proposed schemes were efficient in segmentation, extraction and classification but have a high computational complexity.

The segmentation of the brain tumor is important in the brain tumor diagnosis and treatment. Many techniques were proposed to efficiently segment the brain tumor images, but have certain drawbacks since the tumor cells exhibits different complex characteristics and diverseness in their appearance and boundaries. Among those earlier techniques, automatic tumor segmentation method for MRI images that treated tumor segmentation as a classification problem called the LIPC method [22] was proposed recently. This method classified each voxel into different classes. Thus a new classification framework is obtained by presenting LIPC into the classical classification model. Locality was significant for the calculation of local independent projections for LIPC and also considered in determining whether local anchor embedding is applicable in solving linear projection weights. It also considered the data distribution into different classes by learning a soft max regression model, to improve classification performance. However, there is computational complexity due to dictionary construction and only the patch feature is considered for determining efficiency. Thus, in order to improve the process of segmentation, an efficient framework is presented in this paper.

\section{Proposed methodology}

The proposed technique involves the process of integrated hybrid segmentation approach that combines the features of LIPC, PAM and enhanced possibilistic FCM approach. It classifies the MR images based on local independent projections and used in the partition and determination of the cluster centers. This approach improves the LIPC based 
segmentation by incorporating the classified images as training data. This is used to construct different dictionaries with all classes. The classes are constructed by incorporating additional contextual features to improve the accuracy of the classification. Then, the testing samples are projected to the dictionaries and the reconstruction process is done using local anchor embedding approach.

The segmented image may have complexity due to the intensity deviation of the healthy cells due to tumor mass effect. It deforms the healthy cells bounding the tumor cells that make the intensity of the tumor images to be misled from the accurate boundaries of the tumor region. In order to overcome this problem, intensity adjustment technique is used. In this work, the brain tumor images are split as T1 and $\mathrm{T} 2$ regions and then the segments are combined with synthetic data. Due the intensity adjustment process, the intensities of tumor cells is enhanced greatly than the deformed healthy cell images so that the images are not affected by the changes due to tumor mass effect.

However, in the above work due to the imperfect initialization, the process is only limited to the surface layers and not the layers in depth, which leads to lack of efficient segmentation of the tumor region. This problem is overcome by utilizing an efficient optimization algorithm called ABC Algorithm. In this algorithm, the image segmentation is done by classifying the tumor region into colonies and each colony is enhanced to a solution. The colonies are combined with the synthetic data of the images. The ABC technique enables the segmentation even in deeper layers by partitioning the tumor images around the lesions to increase the efficiency in the segmentation process. Due to the segmentation of images using $\mathrm{ABC}$, the accuracy of the tumor segmentation is also enhanced as the intensity variations are reduced. The smaller colonies each enabled with a solution cause the segmentation of tumor images to be significantly accurate. Figure 1 shows the overall flow diagram of the proposed method.

\subsection{Integrated hybrid segmentation algorithm}

This approach is constructed by combining the advantageous features of the LIPC, and hybrid PAM and EPFCM in order to enhance the accuracy of the segmentation approach.
First, the Gaussian and Poisson noises in the MR Image of the brain are removed using median filter. The irregularities in the de noised image are removed by skull removal technique. The image is then split up into segments in order to construct the dictionary. LIPC method provides the training sample dictionary. This training set is then partitioned into clusters and the cluster centers are obtained using hybrid PAM and EPFCM.

\subsection{Addition of contextual features}

Then the class images are partitioned around the medoids in order to improve the tumor region and also provide accurate segmentation by EPFCM. The local abnormal events (LAE) method is used to reconstruct the dictionaries and training samples. The training samples that are extracted from the original image are fine-tuned by classification based on projection and addition of contextual features.

Incorporating contextual features in the classification improves recognition accuracy. The different types of contextual features used are semantic, spatial and scale context that belongs to categories of probability, position and size respectively. Initially, the global and local contextual feature is extracted for classifying the normal and tumor cells approximately. Then, the three contextual features are applied to these clusters for fine tuning the classification.

The probability or semantic feature is defined for conditional probability that defines the nature of a cell whether it is normal or abnormal. The spatial or the position feature incorporates the position of the cell. When a cell is near the boundary of the tumor region it has higher probability to be a tumor and those far away from boundary has lesser chance. The scale or the size feature of the cells determines the nature of the cell whether it is normal or abnormal.

Thus, the integrated hybrid segmentation approach with contextual features segments the brain tumor effectively. The output of the process that is the segmented image is labeled with $\mathrm{X}$ with scores $\mathrm{Y}$. The segmented image may contain certain amount of healthy cells that may provide an appearance due to the complexity in intensity variations of the tumor cells nearby due to tumor mass effect. This effect deforms the healthy cells that surround the tumor cells. 


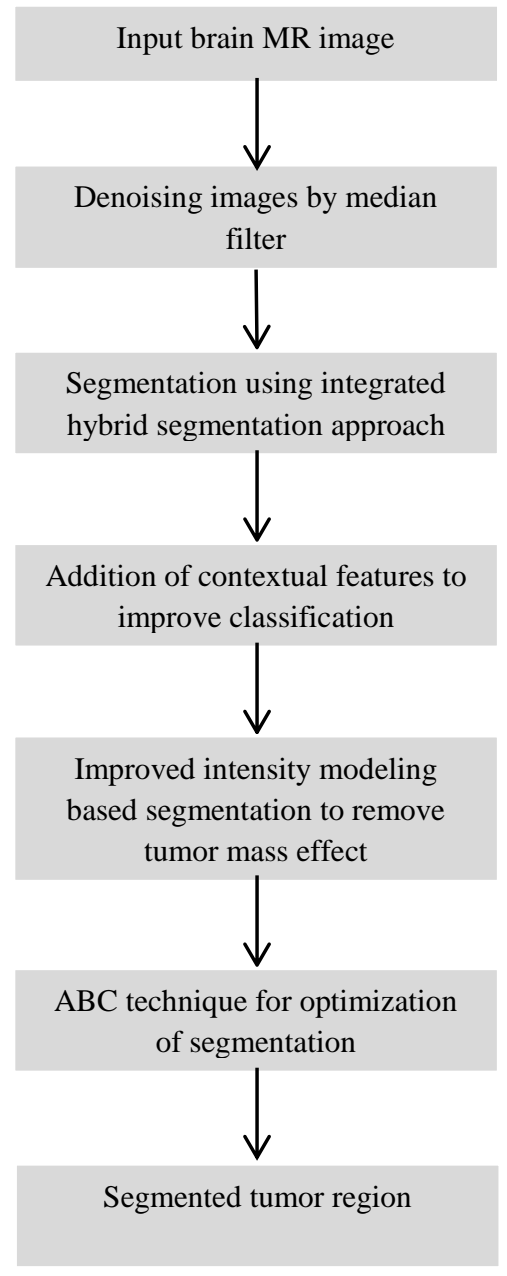

Figure 1 Overall flow diagram of the proposed method

\subsection{Improved intensity modeling based segmentation}

The complexity in the tumor mass effect is solved by utilizing enhanced clustering algorithm with random walker technique. Initially, the segmentation is done using the healthy cell clustering and the outlier detection by multivariate intensity distributions of $\mathrm{T} 1$ and T2 sequences and the constraints impositions where healthy tissue statistics where used as a constraint to improve the accuracy of the segmentation further. Mean, median, average contrast, energy and entropy, skewness and kurtosis are useful first-order statistical features that define the healthy tissue statistics.

\section{Algorithm:}

Input: MRI brain tumor images

Training set $\mathrm{T}=\mathrm{T}_{\mathrm{i}}$, where $\mathrm{I}=1,2 \ldots \mathrm{N}$

Output: Classification score $\mathrm{Y}$ and label of $\mathrm{X}$
1. Initialize the training samples

2. Removal of noise and irregularities in the images.

//hybrid segmentation method

3. Split the image into small subsets for dictionary construction D.

4. Partition the training set into subsets $\mathrm{N}_{\mathrm{i}}$.

//Contextual features

5. The image is clustered initially using global and local contextual features that classify the cells roughly as normal and abnormal.

6. The process of cluster formation is made efficient by adding three more clustering features namely, probability, spatial and size.

7. Compute the cluster center $v_{i}$

8. Calculate the membership value of each cluster.

9. Construct sub dictionaries for each class and reconstruct the dictionary of $\mathrm{T}$ using LAE.

10. Calculate locally linear representation coefficients and vectors for soft regression model.

11. Calculate the reconstruction error for all training sets $\mathrm{T}_{\mathrm{i}}$

12. Testing image is given as input.

13. The image is clustered initially using global and local contextual features and also by three more contextual features that classify the cells roughly as normal and abnormal.

14. Compute the cluster head.

15. Project this model to the dictionary of the training set to identify and estimate the classification score $\mathrm{Y}$ and the reconstruction error for $\mathrm{X}$

16. Achieve the final label of $X$.

17. The segmented image from the MRI image as output.

//Improved intensity modeling based segmentation

18. The segmentation values of the images are denoted as J.

19. Underlying segmentation value is set as $\mathbf{J}_{\mathrm{v}}$

20. The outlier detection is made through the calculation of Manhattan Distance (MD).

21. Each voxels in the MD maps are sampled.

22. Constraints of the healthy cell statistics are imposed such as mean, median, average contrast, energy and entropy, skewness and kurtosis.

23. Include the statistical atlases of healthy cells such as GM, WM and CSF.

24. Acquisition of final seed.

25. Consider the image as a graph and voxels as vertexes connected using edges.

26. Calculate the probability of each voxel.

27. Assign labeling optimization from the arbitrary node to pre- assigned node. 
28. Construction of undirected weighted graph with weighting function as connection between two voxels.

29. $\mathrm{X}_{\text {opt }}$ Optimized label is obtained.

30. Assign highest probability to each vertex is voxels.

31. Resultant segmented image.

3.4Optimized segmentation by $\mathrm{ABC}$ technique The segmentation of the brain MR images is done initially by LIPC hybrid PAM and EPFCM algorithm with additional contextual features, where segmentation is considered as a classification problem. The classification accuracy is further improved by eliminating tumor mass effect by improved intensity modeling. The resulting image gives the segmented image. However, the image segmentation restriction to surface layers is considered for its inaccuracy. Thus, ABC algorithm is implemented for accurate image segmentation. This is performed by classifying the tumor region into colonies and each colony is enhanced to a solution. The overall process is explained as an algorithm given below:

$\mathrm{ABC}$ algorithm is considered for the simulation of intelligent behavior of real honey bees. The algorithm possess three types of bees namely, employed bees, onlooker bees and scout bees in a colony. The number of food sources is same as the number of employed bees, where each bee has a food source. Food sources are selected by the onlooker bees by using a probability and then find new bees around the existing food sources. Scout bee is the employed bee whose food source is abandoned.

Here, this algorithm is used as an optimization for clustering in the segmentation process. The area of the food source is the possible solution of the optimization problem and value of the food source is the value of the problem solutions based on the minimum and maximum amount of the nectar amount in the source ie the fitness function of the cluster center of each cluster.

\section{Algorithm for optimized segmentation by $\mathrm{ABC}$ technique:}

1.Production of food sources with randomly produced cluster centers named as $\mathrm{K}$.

$p_{i j}=p_{j}^{\text {min }}+\operatorname{rand}(0,1)\left(p_{j}^{\max }-p_{j}^{\text {min }}\right)$

Where $i$ is the number of food sources (cluster centers) from 1 to $\mathrm{N}$. and $\mathrm{j}$ is the number of parameters from 1 to $\mathrm{P} . p_{j}^{\text {min }}$ is the minimum and $p_{j}^{\max }$ is the maximum initial values of parameter $\mathrm{P}$.
2.The employed bees detects the cluster center and calculates the fitness function of the clustering problem

$f\left(\operatorname{food}_{i}, d_{i}\right)=w_{1} z_{\max }\left(d, p_{i}\right)+w_{2}\left(d_{\max }-\right.$

$\left.z_{\min }\left(d, p_{i}\right)\right)-w_{3} j_{e}$

Where $d_{\max }$ refers the maximum point of the data and $d$ is related to cluster center. W values are set constant parallel to the literature usage.

3.The fitness functions used here are $j_{e}, z_{\max }$ and $z_{\min }$.

$j_{e}=\sum_{k=1}^{K} \sum_{\forall p \in C_{k}} \frac{z\left(p, m_{k}\right) / n_{k}}{K}$

Where $\mathrm{Ck}$ is the kth cluster, $\mathrm{m}$ is the center and $\mathrm{n}$ is the number of pixels in the cluster, and $\mathrm{z}$ is the Euclidean distance between $\mathrm{p}$ and $\mathrm{m} . \mathrm{p}$ is the pattern of data. $z_{\max }$ and $z_{\min }$ is the maximum and minimum average Euclidean distance respectively. $z_{\text {max }}$ is reduced to satisfy the collection of pixels groups. $z_{\max }$ is maximized to satisfy well separated clusters.

4.If the amount of fitness in new cluster center is greater than the existing cluster center, then employed bee moves to new one or remains in old cluster center.

5.Onlooker bee selects the food source based on the information provided by the employed bee on their fitness.

6.The selection is done by roulette wheel method calculated as

$a_{i}=\frac{\text { fitness }_{i}}{\sum_{i=0}^{N} \text { fitness }_{i}}$

Where fitness $_{i}$ is the fitness of the cluster center.

7.All the onlooker bees have selected the cluster centers.

8.Each onlooker bee computes its fitness by (2).

9.Then new centers are considered and previous ones are discarded.

10.The scout bee produces a new cluster center by (1), when a cluster center cannot be improved further. 11.Accurate segmented tumor image.

Hence, the accuracy of the segmentation process of brain tumor cells from MR images is improved by incorporating hybrid segmentation method with additional contextual features. Then, improved intensity modeling based segmentation method is employed to remove tumor mass effect. In order to solve the problem of inaccuracy of segmentation restricted to surface layers, $\mathrm{ABC}$ optimization algorithm is incorporated.

\section{Results and discussions}

This section shows the results of the existing technique and the various stages of the proposed 
work. The comparison is made between the LIPC technique, integrated hybrid segmentation (LIPCHybrid PAM and EPFCM), integrated hybridintensity modeling based segmentation, and Integrated hybrid-intensity modeled-optimized segmentation by $\mathrm{ABC}$ technique. The comparison is done by the parameters such as dice similarity (DS), Jaccard similarity (JS), accuracy, precision and recall.

\subsection{Dice similarity (DS)}

The DS is a statistical similarity index used to compare similarities between the datasets. It can be evaluated by using the following formula. Let's consider an image of size $m \times n$. $P$ is the voxel set of result while $Q$ is voxel set of ground truth. Then the similarity values can be calculated as, $D S=\frac{2|P \cap Q|}{(|P|+|Q|)}$

\subsection{Jaccard similarity (JS)}

The JS is a statistical similarity index used to compare similarities and diversities between the datasets. It can be evaluated by using the following formula.

$J S=\frac{|P \cap Q|}{|P \cup Q|}$

\subsection{True positive (TP) Rate}

It defines the fraction between number of resulted images having tumor to the total number of images.
$\mathrm{TP}=\frac{\text { No.of resulted image having brain tumor }}{\text { Total no.of images }}$

4.4True negative (TN) rate

It defines the fraction between numbers of images that have no tumor to the total number of images.

$\mathrm{TN}=\frac{\text { No.of Images have no tumor }}{\text { Total no.of images }}$

4.5False positive ( $\mathrm{FP})$ rate

It refers the ratio between number of images that have no tumor but detected as positive to the total number of images.

$\mathrm{FP}=\frac{\begin{array}{c}\text { No.of Images that have no tumor } \\ \text { and detected positive }\end{array}}{\text { Total no.of tumors }}$

4.6False negative $(\mathrm{FN})$ rate

It defines the fraction between number of images that have tumor but detected as negative to the total number of images.

$$
\begin{aligned}
& \text { FN }=\frac{\begin{array}{c}
\text { No.of images that have } \\
\text { tumor and not detected }
\end{array}}{\text { Total no.of tumors }} \\
& \text { Precision }=\frac{T P}{(T P+F P)} \\
& \text { Recall }=\frac{T P}{T P+F N} \\
& \text { Accuracy }=\frac{(T P+F N)}{(T P+T N+F P+F N)}
\end{aligned}
$$

From the Table 1 it is inferred that the proposed integrated hybrid-intensity modeled-optimized segmentation using $\mathrm{ABC}$ technique provides better results when compared to all other techniques used.

Table 1 Comparison results for various segmentation techniques

\begin{tabular}{lllll}
\hline Parameters & $\begin{array}{l}\text { LIPC } \\
\text { Method }\end{array}$ & $\begin{array}{l}\text { Integrated } \\
\text { segmentation (LIPC- hybrid } \\
\text { pam and EPFCM) }\end{array}$ & $\begin{array}{l}\text { hybrid } \\
\text { intensity modeling based } \\
\text { segmentation }\end{array}$ & $\begin{array}{l}\text { Integrated hybrid- } \\
\text { intensity } \\
\text { modeled-optimized segmentation } \\
\text { using abc technique }\end{array}$ \\
\hline DS & 0.6153 & 0.6925 & 0.751 & 0.876 \\
JS & 0.62 & 0.6982 & 0.7611 & 0.8921 \\
Accuracy & 0.70333 & 0.78333 & 0.8200 & 0.90667 \\
Precision & 0.70325 & 0.78333 & 0.81891 & 0.90662 \\
Recall & 0.70339 & 0.78335 & 0.82045 & 0.90676 \\
\hline
\end{tabular}

The graph results that depict the results clearly are given below. Figure 2 clearly shows that the proposed integrated hybrid-intensity modeledoptimized segmentation using $\mathrm{ABC}$ technique has improved values of DS when compared to all other techniques used. Figure 3 clearly shows that the proposed integrated hybrid-intensity modeledoptimized segmentation using $\mathrm{ABC}$ technique has improved values of JS when compared to all other techniques used. Figure 4 clearly shows that the proposed integrated hybrid-intensity modeledoptimized segmentation using $\mathrm{ABC}$ technique has improved values of accuracy rate when compared to all other techniques used. Figure 5 and 6 clearly shows that the proposed integrated hybrid-intensity modeled-optimized segmentation using $\mathrm{ABC}$ technique has increased precision and recall value than other techniques used. 


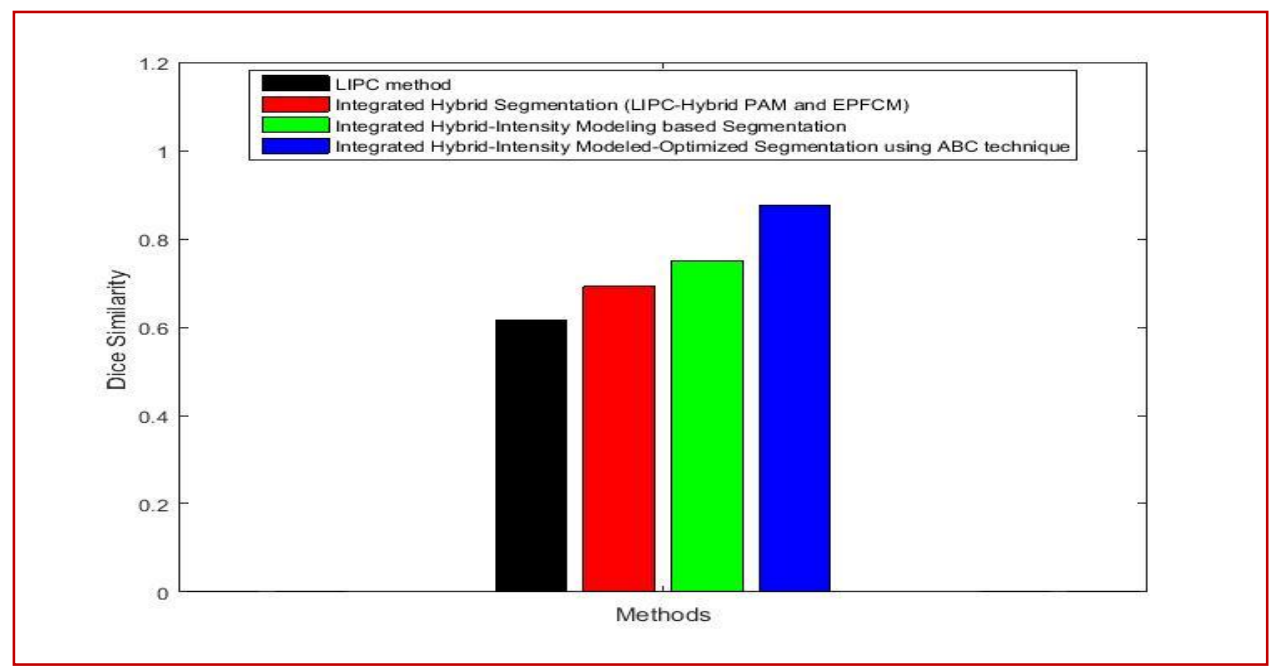

Figure 2 DS value comparison

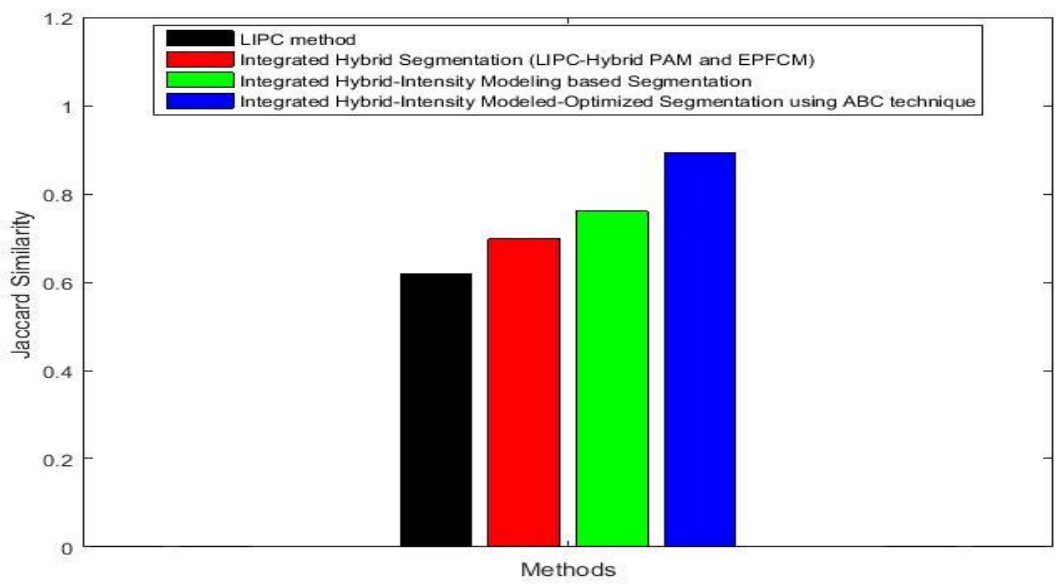

Figure 3 JS value comparison

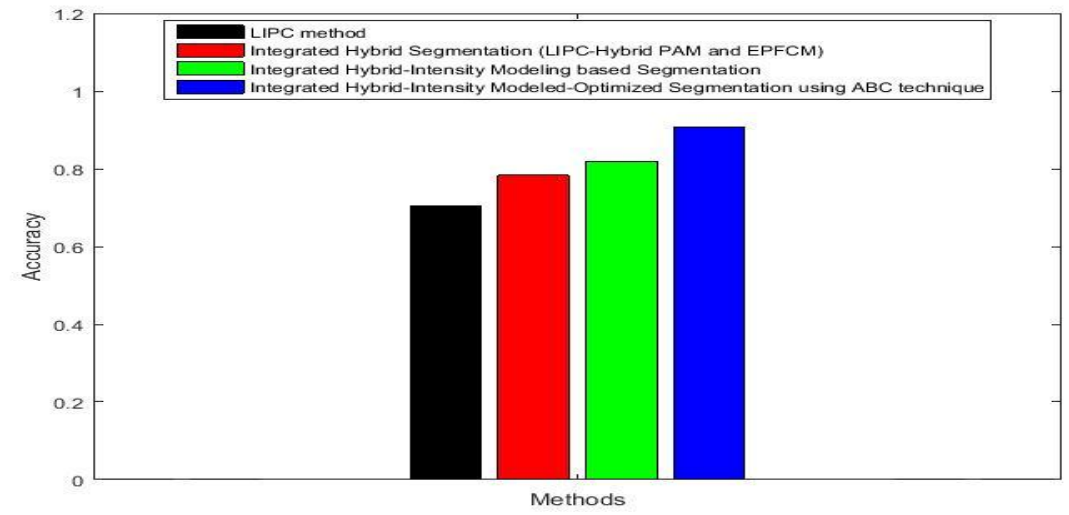

Figure 4 Accuracy value comparison 


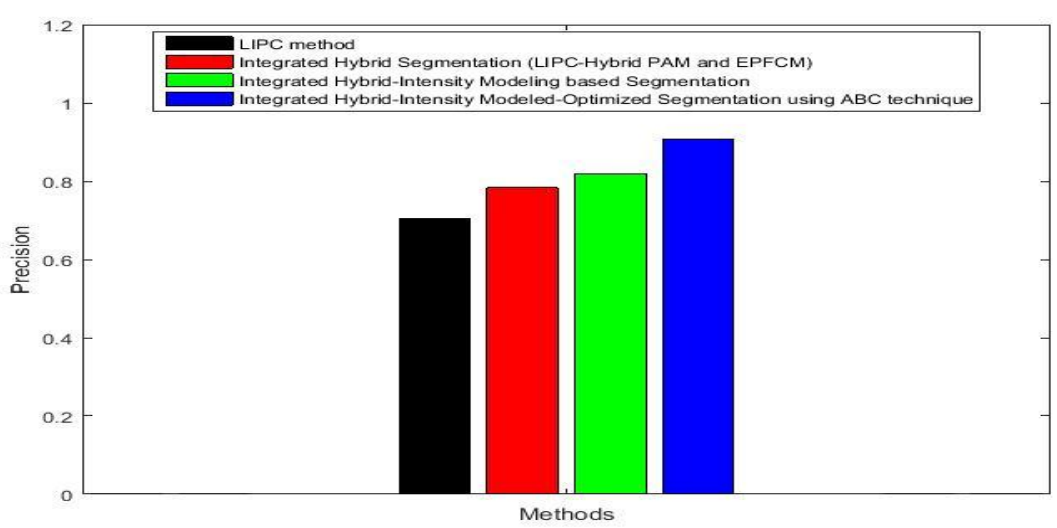

Figure 5 Precision value comparison

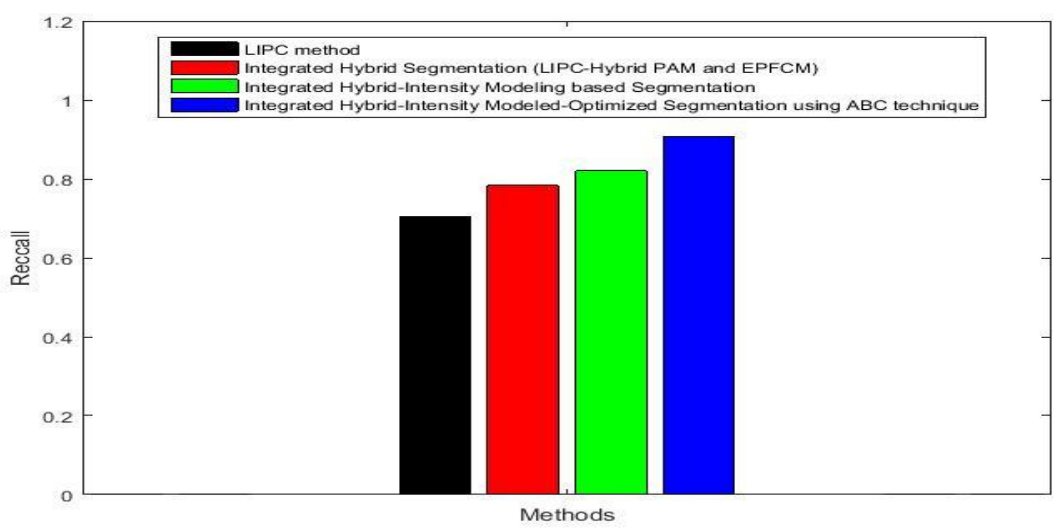

Figure 6 Recall value comparison

\section{Conclusion}

An integrated hybrid- intensity modeled-optimized segmentation using $\mathrm{ABC}$ technique is proposed that incorporates many features for efficient segmentation for the lung tumor. The LIPC method of segmentation is compared with various stages of the proposed technique such as integrated hybridintensity modeled-optimized segmentation using $\mathrm{ABC}$ technique. the proposed segmentation involves various advantages namely, the integrated hybrid segmentation produces efficient and accurate segmented result as contextual features are added to it, then the problem of intensity variations in the normal cells by the tumor mass effect is solved by incorporating intensity modeling based segmentation, then the accuracy of segmentation is more improved by using the ABC algorithm. The experimental results also proved the proposed segmentation technique outperforms all the other existing techniques of lung tumor segmentation. In future, more advanced optimization algorithms could be used to improve the further accuracy on brain tumor segmentation.

\section{Acknowledgment}

None.

\section{Conflicts of interest}

The authors have no conflicts of interest to declare.

\section{References}

[1] Mustaqeem A, Javed A, Fatima T. An efficient brain tumor detection algorithm using watershed \& thresholding based segmentation. International Journal of Image, Graphics and Signal Processing. 2012; 4(10):34-9.

[2] Logeswari T, Karnan M. An enhanced implementation of brain tumor detection using segmentation based on soft computing. In international conference on signal acquisition and processing 2010 (pp. 243-7). IEEE.

[3] Corso JJ, Sharon E, Dube S, El-Saden S, Sinha U, Yuille A. Efficient multilevel brain tumor 
segmentation with integrated Bayesian model classification. IEEE Transactions on Medical Imaging. 2008; 27(5):629-40.

[4] Iftekharuddin KM, Zheng J, Islam MA, Ogg RJ. Fractal-based brain tumor detection in multimodal MRI. Applied Mathematics and Computation. 2009; 207(1):23-41.

[5] Szwarc P, Kawa J, Rudzki M, Pietka E. Automatic brain tumour detection and neovasculature assessment with multiseries MRI analysis. Computerized Medical Imaging and Graphics. 2015; 46:178-90.

[6] Aslam A, Khan E, Beg MS. Improved edge detection algorithm for brain tumor segmentation. Procedia Computer Science. 2015; 58:430-7.

[7] Nabizadeh N, John N, Wright C. Histogram-based gravitational optimization algorithm on single MR modality for automatic brain lesion detection and segmentation. Expert Systems with Applications. 2014; 41(17):7820-36.

[8] Mekhmoukh A, Mokrani K. Improved fuzzy c-means based particle swarm optimization (PSO) initialization and outlier rejection with level set methods for MR brain image segmentation. Computer Methods and Programs in Biomedicine. 2015; 122(2):266-81.

[9] Kanas VG, Zacharaki EI, Davatzikos C, Sgarbas KN, Megalooikonomou V. A low cost approach for brain tumor segmentation based on intensity modeling and $3 \mathrm{D}$ random walker. Biomedical Signal Processing and Control. 2015; 22:19-30.

[10] Forouzanfar M, Forghani N, Teshnehlab M. Parameter optimization of improved fuzzy c-means clustering algorithm for brain MR image segmentation. Engineering Applications of Artificial Intelligence. 2010; 23(2):160-8.

[11] Shanthakumar P, Ganeshkumar P. Performance analysis of classifier for brain tumor detection and diagnosis. Computers \& Electrical Engineering. 2015; 45:302-11.

[12] Ain Q, Jaffar MA, Choi TS. Fuzzy anisotropic diffusion based segmentation and texture based ensemble classification of brain tumor. Applied Soft Computing. 2014; 21:330-40.

[13] Rajendran A, Dhanasekaran R. Fuzzy clustering and deformable model for tumor segmentation on MRI brain image: a combined approach. Procedia Engineering. 2012; 30:327-33.

[14] Gilanie G, Attique M, Naweed S, Ahmed E, Ikram M. Object extraction from T2 weighted brain MR image using histogram based gradient calculation. Pattern Recognition Letters. 2013; 34(12):1356-63.
[15] Wu W, Chen AY, Zhao L, Corso JJ. Brain tumor detection and segmentation in a CRF (conditional random fields) framework with pixel-pairwise affinity and superpixel-level features. International Journal of Computer Assisted Radiology and Surgery. 2014; 9(2):241-53.

[16] Gaonkar B, Macyszyn L, Bilello M, Sadaghiani MS, Akbari H, Attiah MA, et al. Automated tumor volumetry using computer-aided image segmentation. Academic Radiology. 2015; 22(5):653-61.

[17] Joshi A, Charan V, Prince S. A novel methodology for brain tumor detection based on two stage segmentation of MRI images. In international conference on advanced computing and communication systems 2015 (pp. 1-5). IEEE.

[18] Hamamci A, Kucuk N, Karaman K, Engin K, Unal G. Tumor-cut: segmentation of brain tumors on contrast enhanced MR images for radiosurgery applications. IEEE Transactions on Medical Imaging. 2012; 31(3):790-804.

[19] Menon N, Ramakrishnan R. Brain tumor segmentation in MRI images using unsupervised artificial bee colony algorithm and FCM clustering. In international conference on communications and signal processing 2015 (pp. 6-9). IEEE.

[20] Jiang J, Wu Y, Huang M, Yang W, Chen W, Feng Q. 3D brain tumor segmentation in multimodal MR images based on learning population-and patientspecific feature sets. Computerized Medical Imaging and Graphics. 2013; 37(7-8):512-21.

[21] Koley S, Sadhu AK, Mitra P, Chakraborty B, Chakraborty C. Delineation and diagnosis of brain tumors from post contrast T1-weighted MR images using rough granular computing and random forest. Applied Soft Computing. 2016; 41:453-65.

[22] Abdel-Maksoud E, Elmogy M, Al-Awadi R. Brain tumor segmentation based on a hybrid clustering technique. Egyptian Informatics Journal. 2015; 16(1):71-81.

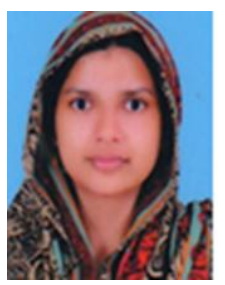

Mubeena achieved the MCA degree from MES Engineering College, Calicut University, India in the year of 2010 and Mphil degree in Computer Science from Bharathiyar University, India in the year of 2017 respectively. Currently, she is a guest lecturer of Computer Science, Farook College, affiliated by Calicut University. She has a total experience of over 3 years. She has published paper on Data mining. Her area of interest is data mining and image processing.

Email: mubeenavallikkatt@gmail.com 